\title{
AN ANALYTICAL MODEL FOR THE DISPERSION OF AIR POLLUTANTS IN A FINITE ATMOSPHERIC BOUNDARY LAYER USING VARIABLE SEPARABLE METHOD
}

\author{
PREM SAGAR BHANDARI ${ }^{\mathbf{~} \boldsymbol{E} \text { VIJAI SHANKER VERMA }}{ }^{\mathbf{2}}$ \\ ${ }^{1}$ Department of Mathematics, Birendra Multiple Campus, Tribhuvan University, Nepal \\ ${ }^{2}$ Department of Mathematics and Statistics D. D. U. Gorakhpur University, Gorakhpur, India
}

\begin{abstract}
We present an analytical model for the dispersion of air pollutants released from a continuous point source in a finite atmospheric boundary layer. In this paper, the wind speed and eddy diffusivity are taken as the power law profile of vertical height functions above the ground as well as the constants. Analytical solutions of the resulting advectiondiffusion equation for these profiles are derived using the variable separable method. It is obtained that there is a good agreement between observed and predicted concentrations given by variable wind speed and eddy diffusivity, but not in between the observed and constant wind speed and eddy diffusivity.
\end{abstract}

KEYWORDS: Concentration of Pollutants, Variable Separable Method, Dispersion, Steady- State Condition.

Received: May 03, 2020; Accepted: May 23, 2020; Published: Jun 09, 2020; Paper Id.: IJMCARJUN20207

\section{INTRODUCTION}

Air pollution is a big problem for people in the world. It has created the environmental problems in every country. The transport of these pollutants can be adequately described by the advection diffusion equation. In the past, several models have been given in finding analytical solutions for the advection-diffusion equation (ADE).

Lebedeff and Hameed have solved the steady-state two-dimensional semi-empirical diffusion equation with wind velocity and eddy diffusivity as power functions of the vertical coordinates by introducing the similarity variable [5]. Naresh and Nath had forwarded an analytical study to examine the characteristics of two-dimensional steady state advective- diffusive transport of air pollutants emitted from ground-based area sources [8].

Sharan and Kumar have given an analytical model for the crosswind integrated concentrations released from a continuous source in a finite atmospheric boundary layer by considering the wind speed as a power law profile of vertical height above the ground and eddy diffusivity as an explicit function of downwind distance from the source and vertical height [10]. Marrouf et al. had given an analytical solution for advection diffusion equation using the wind speed as the linear function of vertical height and eddy diffusivity as variables dependent on vertical height and second as constants [6].

The analytical solutions for advection-diffusion equation for different conditions and cases of the wind speed and eddy diffusivity have been solved by different researchers, Rounds, W. [9], M. Agarwal, Verma, V.S and Srivastava, S. [1], Verma, V.S., Srivastava, U. and Bhandar P.S. [13], Verma, V.S., Srivastava, U. and Bhandar P.S. [14], Sharan and Modani [11], Moreira et al. [7], Kumar, P. and Sharan. M. [4], Costa, C.P. and et al. [2], Verma,V.S.[12], Goncalves, G.A.[3]. 
In this paper, we have formulated an analytical model for the dispersion of air pollutants released from a continuous point source in a finite atmospheric boundary layer. We have taken first the wind speed and eddy diffusivity as the power law profile of vertical height function above the ground and second as the constants. Analytical solutions of the resulting advection-diffusion equation for these profiles of wind speed and eddy diffusivity are obtained. To find the solution, we have used the separation of variables technique. The obtained analytical models are compared with observed data collected from nine experiments conducted at Inshas, Cairo, Egypt.

\section{MATHEMATICAL MODEL}

The dispersion of air pollutants in the finite atmosphere in the rectangular coordinate system is described as follows:

$$
\frac{\partial C}{\partial t}+U \frac{\partial C}{\partial x}+V \frac{\partial C}{\partial y}+W \frac{\partial C}{\partial z}=\frac{\partial}{\partial x}\left(K_{x} \frac{\partial C}{\partial x}\right)+\frac{\partial}{\partial y}\left(K_{y} \frac{\partial C}{\partial y}\right)+\frac{\partial}{\partial z}\left(K_{z} \frac{\partial C}{\partial z}\right)+S
$$

where $\mathrm{C}$ is the mean concentration of a pollutant, $\mathrm{U}, \mathrm{V}$, and $\mathrm{W}$ are the components of the wind speed in $x, y$ and $\mathrm{z}$ directions respectively, $\mathrm{S}$ is the source term, $\left(K_{x}, K_{y}, K_{z}\right)$ are the eddy diffusivity in $x, y$ and $z$ directions respectively.

We take the following assumptions to simplify equation (1).

1) Steady-state conditions so that $\frac{\partial C}{\partial t}=0$

2) Term $W \frac{\partial C}{\partial z}$ is neglected.

3) Term $V \frac{\partial C}{\partial y}$ is neglected.

4) Source (physical/chemical) pollutants are ignored so that $S=0$.

Applying the above assumptions, equation (1) becomes:

$$
U \frac{\partial C}{\partial x}=\frac{\partial}{\partial x}\left(K_{x} \frac{\partial C}{\partial x}\right)+\frac{\partial}{\partial y}\left(K_{y} \frac{\partial C}{\partial y}\right)+\frac{\partial}{\partial z}\left(K_{z} \frac{\partial C}{\partial z}\right)
$$

As the advection term in $x$ - direction is larger than the diffusion in $x$-direction, therefore we neglect the diffusion term in $x$-direction. Thus, we have

$$
U \frac{\partial C}{\partial x}=\frac{\partial}{\partial y}\left(K_{y} \frac{\partial C}{\partial y}\right)+\frac{\partial}{\partial z}\left(K_{z} \frac{\partial C}{\partial z}\right)
$$

We solve (3) under the following boundary conditions:

$$
\begin{aligned}
& \frac{\partial C(x, z)}{\partial z}=0 \text { at } \mathrm{z}=0, \mathrm{z}=\mathrm{h} \\
& C(x, y, z)=0 \text { at } \mathrm{z}=\mathrm{h} \\
& U C=Q \delta\left(z-z_{s}\right), \text { at } \mathrm{x}=0 \\
& C(x, y, z)=0 \text { as } x, y, z \rightarrow \infty
\end{aligned}
$$

where $\delta$ is the Dirac's delta function.

Condition (4) states that the pollutant is assumed to be a perfectly total absorption. Condition (5) states that the pollutant is totally penetrated through the top of the inversion/mixed layer located at height h. Condition (6) states that a 
continuous point source with strength $\mathrm{Q}$ is assumed to be located at $(0, y, z)$ and condition (7) states that far away from the source, the concentration decreases to zero.

\section{METHOD OF SOLUTION}

We solve the above problem under two cases:

\section{Case I: Case of Variable Eddy Diffusivity and Variable Wind Speed.}

For this case, we define wind speed and eddy diffusivity as:

$$
\begin{aligned}
& U=u_{1} z^{n}, z \neq 0 \text { and } U=u_{0} \text { at } z=0 \\
& \text { and } K_{z}=u_{2} z^{n}
\end{aligned}
$$

where $u_{1}$ is the friction velocity and $u_{2}$ is the turbulence intensity.

Integrating equation (3) with respect to y from $-\infty$ to $\infty$, we get

$$
U \frac{\partial}{\partial x} \int_{-\infty}^{\infty} C(x, y, z) d y=\int_{-\infty}^{\infty} \frac{\partial}{\partial y}\left(K_{y} \frac{\partial C(x, y, z)}{\partial y}\right) d y+\frac{\partial}{\partial z}\left[K_{z} \frac{\partial}{\partial z} \int_{-\infty}^{\infty} C(x, y, z) d y\right]
$$

Now, for simplicity, let us assume

$$
\int_{-\infty}^{\infty} C(x, y, z) d y=C_{y}(x, z) \text { and } \int_{-\infty}^{\infty} \frac{\partial}{\partial y}\left(K_{y} \frac{\partial C(x, y, z)}{\partial y}\right) d y=0
$$

Then, the equation (10) reduces to $U \frac{\partial}{\partial x} C_{y}(x, z)=\frac{\partial}{\partial z}\left[K_{z} \frac{\partial}{\partial z} C_{y}(x, z)\right]$

Using equations (8) and (9) in equation (11), we get

$$
\begin{aligned}
& u_{1} z^{n} \frac{\partial}{\partial x} C_{y}(x, z)=\frac{\partial}{\partial z}\left[u_{2} z^{n} \frac{\partial}{\partial z} C_{y}(x, z)\right] \\
& \frac{\partial C_{y}}{\partial x}=\frac{u_{2} n}{u_{1}} z^{-1} \frac{\partial C_{y}}{\partial z}+\frac{u_{2}}{u_{1}} \frac{\partial^{2} C_{y}}{\partial z^{2}}
\end{aligned}
$$

We solve the equation (13) analytically by using the variable separable method.

Therefore, we assume the solution of the equation (13) in the following form:

$$
C_{y}(x, z)=X(x) Z(z)
$$

Differentiating (14) with respect to $x$ and $z$ partially, we get

$$
\frac{\partial C_{y}}{\partial x}=\frac{\partial X}{\partial x} Z, \frac{\partial C_{y}}{\partial z}=\frac{\partial Z}{\partial z} X \text { and } \frac{\partial^{2} C_{y}}{\partial z^{2}}=X \frac{\partial^{2} Z}{\partial z^{2}}
$$

Using these values in equation (13), we get

$\frac{\partial X}{\partial x} Z=\frac{u_{2} n}{u_{1}} Z^{-1} X \frac{\partial Z}{\partial z}+\frac{u_{2}}{u_{1}} X \frac{\partial^{2} Z}{\partial z^{2}} \quad$ or $\quad \frac{1}{X} \frac{\partial X}{\partial x}=\frac{u_{2} n}{u_{1}} \frac{1}{z} \frac{\partial Z}{\partial z}+\frac{u_{2}}{u_{1}} \frac{1}{z} \frac{\partial^{2} Z}{\partial z^{2}}=-\lambda^{2}$

where $\lambda^{2}$ is a constant.

Taking the first and last ratios of equation (15), we get $\frac{1}{X} \frac{\partial X}{\partial x}=-\lambda^{2}$ 
The general solution of equation (16) is given by $X(x)=\gamma e^{-\lambda^{2} x}$

where $\gamma$ is a constant.

Taking the second and last ratios of equation (15), we get

$\frac{1}{z}\left(\frac{u_{2} n}{u_{1}} \frac{\partial Z}{\partial z}+\frac{u_{2}}{u_{1}} \frac{\partial^{2} Z}{\partial z^{2}}\right)=-\lambda^{2}$

Suppose $\alpha=\frac{u_{2}}{u_{1}}$ and $\beta=\frac{u_{2} n}{u_{1}}$ in equation (18), therefore, we get

$$
z \frac{\partial^{2} Z}{\partial z^{2}}+n \frac{\partial Z}{\partial z}+\frac{\lambda^{2}}{\alpha} z Z=0, \text { since } \frac{\beta}{\alpha}=n
$$

Now, let us put $p=Z \sqrt{Z}$ so that $Z=\frac{p}{\sqrt{z}}$

Differentiating equation (20) with respect to $\mathrm{z}$, we get

$$
\frac{\partial Z}{\partial z}=z^{-\frac{1}{2}} \frac{\partial p}{\partial z}-\frac{p}{2} z^{-\frac{3}{2}}
$$

Again differentiating (21) with respect to z, we get

$$
\frac{\partial^{2} Z}{\partial z^{2}}=z^{-\frac{1}{2}} \frac{\partial^{2} p}{\partial z^{2}}-z^{-\frac{3}{2}} \frac{\partial p}{\partial z}+\frac{3 p}{4} z^{-\frac{5}{2}}
$$

Using the equations (20)-(22) in equation (19) and simplifying,we get

$$
z^{2} \frac{d^{2} p}{d z^{2}}+(n-1) z \frac{d p}{d z}+\left(z^{2} \frac{\lambda^{2}}{\alpha}-\frac{3-2 n}{4}\right) p=0
$$

To solve the above equation, we let $\frac{z \lambda}{\sqrt{\alpha}}=t$ so that $\frac{d t}{d z}=\frac{\lambda}{\sqrt{\alpha}}$

Now, we have $\frac{d p}{d z}=\frac{d p}{d t}\left(\frac{d t}{d z}\right)=\frac{d p}{d t}\left(\frac{\lambda}{\sqrt{\alpha}}\right)$

$$
\text { which implies that } \quad \frac{d}{d z}=\frac{\lambda}{\sqrt{\alpha}} \frac{d}{d t}
$$

Also, we have $\frac{d^{2} p}{d z^{2}}=\frac{d}{d z}\left(\frac{d p}{d z}\right)=\frac{\lambda}{\sqrt{\alpha}} \frac{d}{d t}\left(\frac{\lambda}{\sqrt{\alpha}} \frac{d p}{d t}\right)$

$$
\text { which implies that } \quad \frac{d^{2} p}{d z^{2}}=\frac{\lambda^{2}}{\alpha} \frac{d^{2} p}{d t^{2}}
$$

Using the equation (24)-(27) in equation (23) and simplifying,we get

$$
t^{2} \frac{d^{2} p}{d t^{2}}+(n-1) t \frac{d p}{d t}+\left(t^{2}+\frac{3-2 n}{4}\right) p=0
$$

Now taking $n=2$ in equation (28) as a particular case, we get

$$
t^{2} \frac{d^{2} p}{d t^{2}}+t \frac{d p}{d t}+\left\{t^{2}-(1 / 2)^{2}\right\} p=0
$$

which is a Bessel differential equation of order $1 / 2$.

The solution of equation (29) is given by

$$
p=A J_{1 / 2}(t)+B J_{-1 / 2}(t)
$$


where $J_{1 / 2}$ and $J_{-1 / 2}$ are Bessel functions of first kind of order $1 / 2$ and $\mathrm{A}$ and $\mathrm{B}$

are some constants.

Using the equations (24) and (20) in equation (30), we get

$$
Z \sqrt{z}=A J_{1 / 2}\left(\frac{z \lambda}{\sqrt{\alpha}}\right)+B J_{-1 / 2}\left(\frac{z \lambda}{\sqrt{\alpha}}\right)
$$

Therefore, we have $Z=z^{-\frac{1}{2}}\left[A J_{1 / 2}\left(\frac{z \lambda}{\sqrt{\alpha}}\right)+B J_{-1 / 2}\left(\frac{z \lambda}{\sqrt{\alpha}}\right)\right]$

Using the conditions (4) and (5) along with equation (12), we get

$$
Z=0 \text { at } z=0, z=h
$$

Using the condition (32) at $z=0$ in equation (31), we get $B=0$

Again, using condition (32) at $=h$, we get

$$
h^{-\frac{1}{2}} J_{1 / 2}\left(\frac{h \lambda}{\sqrt{\alpha}}\right)=0 \text { or } \quad h^{-\frac{1}{2}} J_{1 / 2}(\eta h)=0, \text { where } \eta=\frac{\lambda}{\sqrt{\alpha}}
$$

Equation (33) represents Strom-Liouville Eigen value problem which have the following corresponding Eigen functions:

$$
Z_{\alpha}(z)=z^{-\frac{1}{2}} J_{1 / 2}\left(\eta_{\alpha} z\right) ; \alpha=1,2,3, \ldots \ldots
$$

Therefore, the general solution of equation (12) is obtained by using equations (17) and (34) as:

$$
C_{y}(x, z)=z^{-1 / 2}\left[\sum_{\alpha=1}^{\infty} A_{\alpha} J_{1 / 2}\left(\eta_{\alpha} z\right) e^{-\lambda^{2}}\right]
$$

where $A_{\alpha}, \alpha=1,2,3, \ldots \ldots \infty$ are the unknown coefficients. Equation (35) represents the concentration distribution $C_{y}$ through the Fourier-Bessel series corresponding to a set of Eigen function $Z_{\alpha}$.

\section{Estimation of the Coefficient $A_{\alpha}$ for Crosswind Integrated Concentration}

If the source is at $x=0$, then equation (6) gives:

$u_{1} z^{3 / 2}\left[\sum_{\alpha=1}^{\infty} A_{\alpha} J_{1 / 2}\left(\eta_{\alpha} z\right)\right]=Q_{p} \delta\left(z-h_{s}\right)$

To find the value of $A_{\alpha}$, we use orthogonality of Eigen functions series and we obtain

$$
A_{\alpha}=\frac{2 Q_{p} z_{s}^{3 / 2} J_{1 / 2}\left(\eta_{\beta} z_{S}\right)}{u_{1} h^{2}\left[J_{1 / 2}+1\right.}
$$

Substituting $A_{\alpha}$ in equation (35), the final solution is given by

$$
\begin{aligned}
& C_{y}(x, z)=\frac{2 Q_{p} z^{-1 / 2} z_{s}^{3 / 2} J_{1 / 2}\left(\eta_{\beta} z_{S}\right)}{u_{1} h^{2}\left[J_{1 / 2+1}\left(\eta_{\beta} h\right)\right]^{2}}\left[\sum_{\alpha=1}^{\infty} J_{1 / 2}\left(\eta_{\alpha} z\right)\right] e^{-\lambda^{2} x} \\
& \text { in which } \eta_{\beta} h \text { is given by } J_{1 / 2}\left(\eta_{\beta} h\right)=0
\end{aligned}
$$




\section{Case 2:Case of Constant Eddy Diffusivity and Constant Wind Speed.}

For this case, we take $K_{z}=k=$ a constant and $\mathrm{U}=$ constant.

Now, integrating equation (3) with respect to y from $-\infty$ to $\infty$, we get

$U \frac{\partial C_{y}(x, z)}{\partial x}=K_{z} \frac{\partial^{2} C_{y}(x, z)}{\partial z^{2}}$

$\frac{\partial C_{y}(x, z)}{\partial x}=\frac{k}{U} \frac{\partial^{2} C_{y}(x, z)}{\partial z^{2}}$

We solve the partial differential equation (41) analytically by the separation of variables technique. We assume the solution of equation (41) in the form:

$C_{y}(x, z)=X(x) Z(z)$

Now, differentiating (42) with respect to $x$ and $z$ partially, we get

$\frac{\partial C_{y}}{\partial x}=\frac{\partial X}{\partial x} Z, \frac{\partial C_{y}}{\partial z}=X \frac{\partial Z}{\partial z}$ and $\frac{\partial^{2} C_{y}}{\partial z^{2}}=X \frac{\partial^{2} Z}{\partial z^{2}}$

Using the above values in equation (41), we get

$\frac{\partial X}{\partial x} Z=\frac{K X}{U} \frac{\partial^{2} Z}{\partial z^{2}}$ or $\frac{1}{X} \frac{\partial X}{\partial x}=\left(\frac{K}{U}\right) \frac{1}{Z} \frac{\partial^{2} Z}{\partial z^{2}}=-\lambda^{2}$, where $\lambda^{2}$ is a constant.

Using the first and last ratios of equation (43), we get $\quad \frac{1}{X} \frac{\partial X}{\partial x}=-\lambda^{2}$

Using the second and last ratios of equation (43), we get $\left(\frac{K}{U}\right) \frac{1}{Z} \frac{\partial^{2} Z}{\partial z^{2}}=-\lambda^{2}$

The general solution of equation (44) is given by $X(x)=\gamma e^{-\lambda^{2} x}$

Equation (45) can be re-written as

$\left(\frac{K}{U}\right) \frac{\partial^{2} Z}{\partial z^{2}}+\lambda^{2} Z=0$

The solution of equation (47) is given as:

$Z(z)=A \cos \left(\lambda \sqrt{\frac{U}{k}}\right) z+B \sin \left(\lambda \sqrt{\frac{U}{k}}\right) z$

where $A$ and $B$ are constants.

Using the equations (46) and (48) in equation (42), we get solution as:

$C_{y}(x, z)=e^{-\lambda^{2} x}\left[A \cos \left(\lambda \sqrt{\frac{U}{K}}\right) z+B \sin \left(\lambda \sqrt{\frac{U}{K}}\right) z\right]$

Differentiating equation (49) with respect to $z$, we get

$\frac{\partial C_{y}(x, z)}{\partial z}=e^{-\lambda^{2} x}\left[-A \lambda \sqrt{\frac{U}{K}} \sin \left(\lambda \sqrt{\frac{U}{K}}\right) z+B \lambda \sqrt{\frac{U}{K}} \cos \left(\lambda \sqrt{\frac{U}{K}}\right) z\right]$

Applying condition (4) on (50), we get $B=0$ and so equation (49) becomes:

$C_{y}(x, z)=e^{-\lambda^{2} x} A \cos \left(\lambda \sqrt{\frac{\bar{U}}{K}}\right) z$ 
Again, applying the boundary condition (6) in equation (51), we get

$A=\frac{Q}{U_{z_{S}}} \sec \left(\lambda \sqrt{\frac{U}{K}}\right) z_{S}$, where $U_{z_{S}}$ be the wind speed at $z_{s}$.

Using the value of $A$ in equation (51), we get

$$
C_{y}(x, z)=\frac{Q}{U_{z_{s}}} e^{-\lambda^{2} x} \cos \left(\lambda \sqrt{\frac{U}{K}}\right) z \sec \left(\lambda \sqrt{\frac{U}{K}}\right) z_{s}
$$

\section{RESULTS AND DISCUSSION}

The concentration of pollutants was computed using the data collected at vertical distance of a $30 \mathrm{~m}$ multi-level micrometeorological tower. Table 1 gives the predicted concentrations using the analytical solution equations (38) and (53) and the observed concentrations for I isotope tracer nine experiments in moderate wind with unstable conditions at Inshas Cairo, Egypt as below:

Table 1: Observed (For Nine Experiments) and Predicted Concentrations from Equations (38) and (53).

\begin{tabular}{|c|c|c|c|c|c|}
\hline $\begin{array}{c}\text { Test } \\
\text { Numbers }\end{array}$ & $\begin{array}{c}\text { Downwind } \\
\text { Distance }\end{array}$ & $\begin{array}{c}\text { Vertical } \\
\text { Distance }\end{array}$ & $\begin{array}{c}\text { Observed } \\
\text { Concentration }\end{array}$ & $\begin{array}{c}\text { Predicted } \\
\text { Concentration } \\
\text { Equation(38) }\end{array}$ & $\begin{array}{c}\text { Predicted } \\
\text { Concentration } \\
\text { Equation(53) }\end{array}$ \\
\hline 1 & 100 & 5 & .025 & .043 & .032 \\
\hline 2 & 98 & 10 & .037 & .057 & .033 \\
\hline 3 & 115 & 5 & .091 & .066 & .090 \\
\hline 4 & 135 & 5 & .197 & .130 & .148 \\
\hline 5 & 99 & 2 & .272 & .216 & .155 \\
\hline 6 & 184 & 11 & .188 & .108 & .162 \\
\hline 7 & 165 & 12 & .447 & .320 & .032 \\
\hline 8 & 134 & 7.5 & .123 & .110 & .033 \\
\hline 9 & 96 & 5 & .032 & .037 & .032 \\
\hline
\end{tabular}

Figure 1 shows that the variation of predicted and observed concentration of nine typical tests with downwind distance. It is seen that there is good agreement between observed and predicted concentration given by equation (38) than predicted concentration given by equation (53).

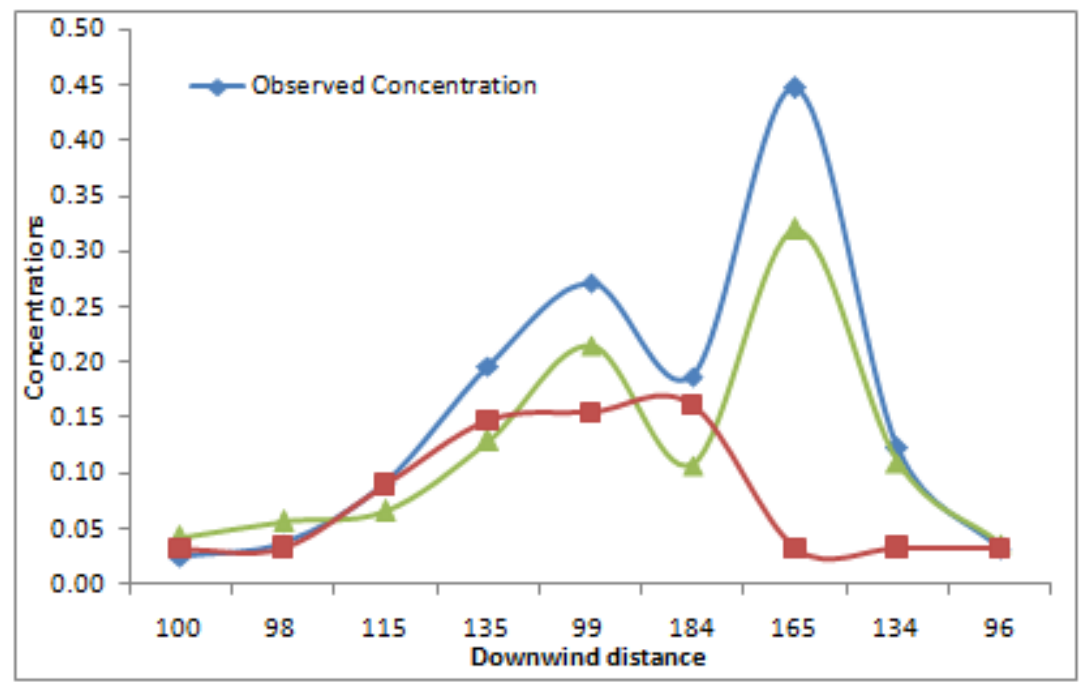

Figure 1: Comparison among the Observed and Two Predicted Concentrations 
Figure 2 shows that there is good agreement between predicted concentration from equation (38) and observed concentration.

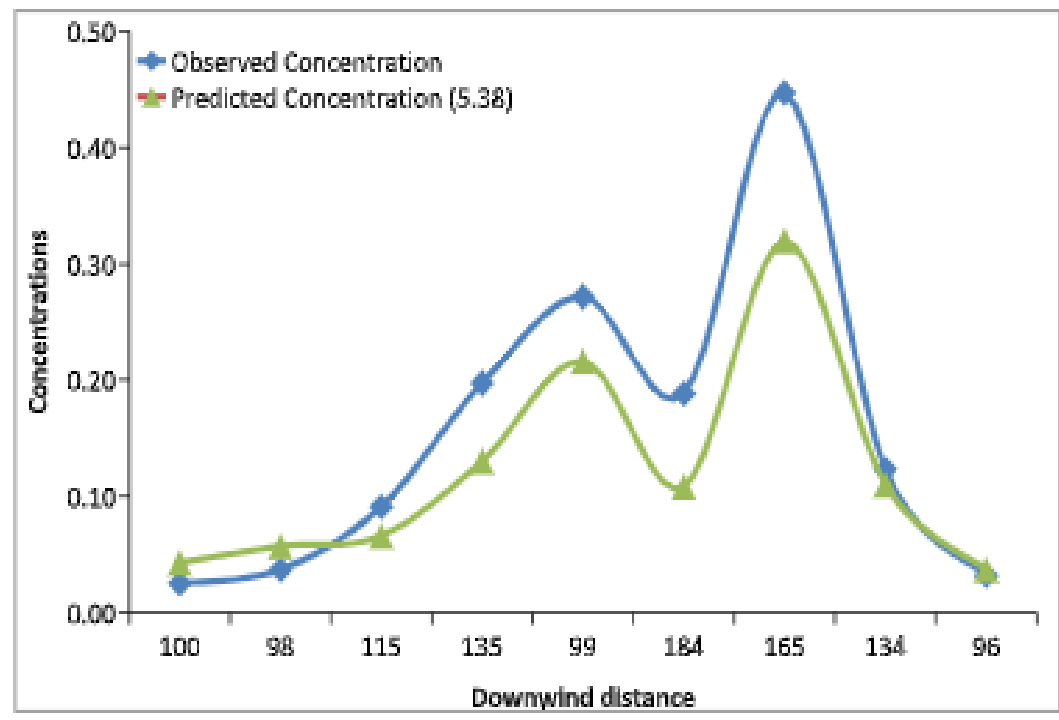

Figure 2: Comparison between the Observed and Predicted Concentration (38).

Figure 3 shows that there is no good agreement between predicted concentration from equation (53) and observed concentration.

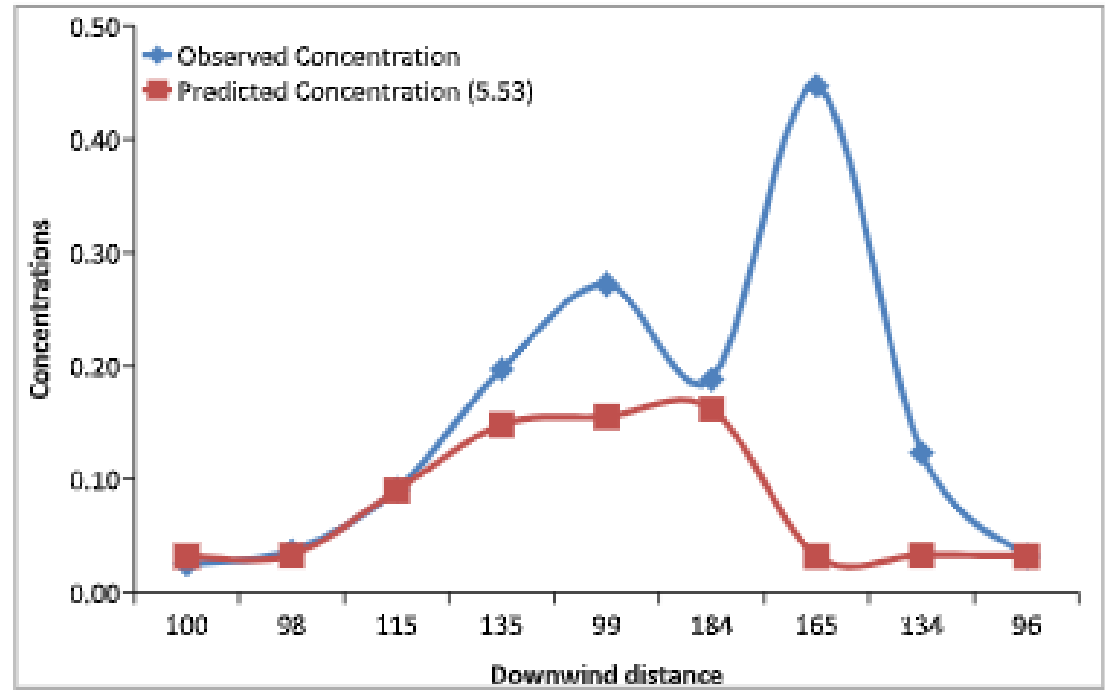

Figure 3: Comparison between the Observed and Predicted Concentration (53).

\section{CONCLUSIONS}

In this paper, a mathematical model for dispersion of air pollutants is formulated in moderate winds. The wind speeds and eddy diffusivities are taken as constants in the first case and variables in the second case. The obtained analytical results are compared with observed data collected from nine experiments conducted at Inshas, Cairo, Egypt. From the graph, it is found that there is a good agreement between observed and predicted concentration given by variable wind speed and eddy diffusivity equation (38) than predicted concentration given by constant wind speed and eddy diffusivity equation (53). 


\section{REFERENCES}

1. Agarwal, M., Verma, V. S. and Srivastava, S. (2008) “An analytical approach to the problem of dispersion of an air pollutant with variable wind velocity," J. Nat. Acad. Math., 22 pp. 51-62.

2. Costa, C.P., Vilhena, M.T., Moreina D.M. and Tirabassi, T. (2006).Semi-analytical solution of the steady three-dimensional advection- diffusion equation in the planetary boundary layer. Atmospheric environment 40, 5659-5669.

3. Goncalves, G. A., Regis, S. and Buske, D. (2013).An analytical formulation for pollutants dispersion simulation in the atmospheric boundary layer. Journal of Environmental Protection, 4, 57-64.

4. Kumar, P. and Sharan, M.(2016).An analytical dispersion model for sources in the atmospheric surface layer with dry deposition to the ground surface. Aerosol and Air Quality Research,16,1284-1293.

5. Lebedeff, S.A. and Hameed S. (1975). Steady state solution of the semi-empirical diffusion equation for area sources. 14,546549.

6. Marrouf, A.A., Khaled,E., Maha, S., Mohamed, A.S. and Ismail, G.(2015). The influence of eddy diffusivity variation on the atmospheric diffusion equation. 4, 109-118.

7. Moreira, D.M., Villena, M.T., Tirabassi, T., Buske, D. and Cotta, R. (2005). Near-source atmospheric pollutant dispersion using the new GILTT method. Atmos. Environ. 39, 6289-6294.

8. Naresh, M. and Nath, M. (1990). The effects of chemical reaction on the dispersion of air pollutants over area sources. Kluwer Academic publishers, 99-111.

9. Rounds, W.(1955). Solution of two dimensional equation.Trans.Atmos. Geophy.Union,36,395-405.

10. Sharan, M. and Kumar, P. (2009). An analytical model for crosswind integrated concentrations released from a continuous source in a finite atmospheric boundary layer. Atmospheric Environment, 43, 2268-2277.

11. Sharan, M. and Modani, M. (2006). A two-dimensional analytical model for the dispersion of air-pollutants in the atmosphere with a capping inversion. Atmos. Environ. 40, 3469-3489.

12. Verma, V.S. (2011).An analytical approach to the problem of Dispersion of an air pollutant with constant wind velocity and constant removal rate. Journal of international academy of physical sciences, 15, 43-50.

13. Verma, V.S. Srivastava, U. and Bhandari, P.S. (2015), A mathematical model on dispersion of air pollutants. Intenational Journal of Science and Research, 4, 1904-1907.

14. Verma, V.S. Srivastava, U. and Bhandari, P.S. (2016) An analytical approach to a problem on dispersion of air pollutants. International Journal of Recent Scientific Research, 7, 10, 13850-13857. 
